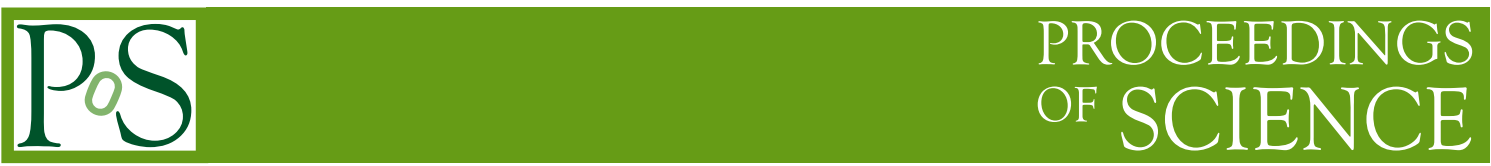

\title{
Jet results in heavy-ion collisions from CMS
}

\author{
P. K. Netrakanti (for the CMS Collaboration) * \\ Bhabha Atomic Research Center, Mumbai-400084, India \\ E-mail: pawannetrakanti@gmail.com
}

\begin{abstract}
Jet production in heavy-ion collisions at relativistic energies provide an excellent opportunity to study the in-medium modification and the energy loss mechanism of partons in strongly interacting quark-gluon plasma. A detailed overview of jet results from CMS experiment for $\mathrm{PbPb}, \mathrm{pp}$ and $\mathrm{pPb}$ collisions are presented in this paper. The in-medium modification of the jets are quantified with the isolated photon-jet imbalance measurements in the $\mathrm{PbPb}$ collisions. Comparison of nuclear modification factor $\left(R_{A A}\right)$ for different particles, inclusive jets and $\mathrm{b}$-jets are presented for $\mathrm{PbPb}$ and $\mathrm{pPb}$ collisions at $2.76 \mathrm{TeV}$ and $5.02 \mathrm{TeV}$, respectively. The differential jet shapes and jet fragmentation functions are presented for the charged particles in $\mathrm{pp}$ and $\mathrm{PbPb}$ collisions. The momentum flow of charged particles in unbalanced jet events is discussed in context of recovery of the lost energy by these energetic jets in the $\mathrm{PbPb}$ collisions.
\end{abstract}

7th International Conference on Physics and Astrophysics of Quark Gluon Plasma

1-5 February, 2015

Kolkata, India

${ }^{*}$ Speaker. 


\section{Introduction}

Heavy-ion collisions at ultra relativistic energies provide the opportunity to study the properties of strongly interacting matter at extreme temperatures and energy densities. The highly energetic partons produced by hard scattering in these collisions loose energy while traversing through the hot and dense medium formed in the collisions [1]. This energy loss of partons was experimentally observed in the suppression of yields of particles with high transverse momentum $\left(p_{T}\right)$ in the most central AuAu collisions as compared to pp collisions at Relativistic Heavy Ion Collider (RHIC) experiments $[2,3,4,5]$. The suppression factor was almost an order of 5 times in most central collisions and starts to reduce from central to peripheral collisions. The suppression pattern was not observed in the controlled experiment at RHIC using the $d+\mathrm{Au}$ collisions at $\sqrt{s_{N N}}=200$ $\mathrm{GeV}$. However most of these studies at RHIC were only limited to the single particle level and could not be performed with the full jet reconstruction. The begining of $\mathrm{pp}$ and $\mathrm{PbPb}$ collisions at Large Hadron Collider (LHC), the Compact Muon Solenoid (CMS) experiment has demonstrated the capabilities of full jet reconstruction in these high energy collisions. With an excellent set of detectors, CMS experiment can identify and measure charged hadrons, neutral hadrons, electrons, muons and photons upto large pseudorapidity $(\eta)$ and $p_{T}$ ranges [6]. These particles are used to reconstruct jets with appropriate clustering algorithms which would be discussed briefly later in the proceedings.

The most central $\mathrm{PbPb}$ collisions are those that have the highest nuclear overlap and are represented by the lowest centrality percentiles. The momentum asymmetry $\left(A_{J}=\frac{\left(p_{T, 1}-p_{T, 2}\right)}{\left(p_{T, 1}+p_{T, 2}\right)}\right)$ in dijets has been successfully demonstrated in most central $\mathrm{PbPb}$ collisions compared to pp collisions [7]. This large asymmetry in jet yields for central $\mathrm{PbPb}$ collisions indicate the energy loss of partons while propagating through medium. However, there was no azimuthal angular decorrelation of jet pairs was observed which lead to the conclusion that the energy loss by these partons is not enough to cause deviations in the azimuthal angles while passing through the medium. To quantify these dijet results, CMS has performed an extensive study of isolated photon jet events in the $\mathrm{PbPb}$ collisions and compared them to baseline obtained from PYTHIA and pp data at the same colliding energy. To recover the lost energy of these jets, the momentum flow of particles around the jet axis was done by including lower momentum particles in the extended reach of larger jet-radii in $\eta-\phi$ phase space. The difference in the momentum flow carried by charged particles near the dijet axis are measured and compared between $\mathrm{pp}$ and $\mathrm{PbPb}$ collisions. Furthermore, the energy loss of jets in heavy-ion collisions is expected to have flavor dependence on the originating partons from which jet are produced by fragmentation. Jets from heavy quark are expected to radiate less energy due to the dead cone effect and should be least modified or loose energy in the hot and dense medium [8]. Most of the jets produced in high energy collisions mainly originate from light flavor quarks ( $u$ and d) and gluons. Occasionally a heavy quark ( $c$ or b) are produced and are associated with the jet production. Though these processes are rare, the high luminosity delivered by LHC and efficient trigger and discriminating software algorithms allow us to reconstruct $\mathrm{b}$-jets in $\mathrm{PbPb}$ collisions. The experimental determination of $b$-jet production cross-sections would highly constrain the energy loss models and also provide a direct connection to the initial b-quark fragmentation. All the results described in proceeding use collisions of pp at $\sqrt{s}=2.76 \mathrm{TeV}$ with integrated luminosity of $5.3 \mathrm{pb}^{-1}, \mathrm{PbPb}$ at $\sqrt{s_{N N}}=2.76 \mathrm{TeV}$ with integrated luminosity of $150 \mu b^{-1}$ and $\mathrm{pPb}$ at $\sqrt{s_{N N}}=$ 


\subsection{TeV with integrated luminosity of $35 n b^{-1}$.}

\section{Jet reconstruction}

Jets are reconstructed using the CMS particle flow algorithm [9] which attempts to combine all stable particles in an event from various sub-detector systems. These particle flow objects are then clustered using the anti- $k_{T}$ [10] algorithm, with a resolution parameter of 0.3 , encoded in the FastJet [11] framework. In $\mathrm{pPb}$ and $\mathrm{PbPb}$ jet events the underlying event is removed with the iterative pileup subtraction technique [12]. The b-jets are tagged by using a discriminator which uses the information of the flight distance of the reconstructed secondary vertices with respect to the nominal collision interaction point. This discriminator is called Simple Secondary Vertex tagger (SSV) [13], and is based on the flight distance significance (flight distance divided by its uncertainty) of reconstructed secondary vertices. For the analysis of $\mathrm{PbPb}$ dijet momentum flow, the underlying event contribution was estimated and removed using the HF/Vornoi technique [14]. This technique uses the transverse energy azimuthal profile from the Hadron Forward (HF) detector to subtract the underlying event contribution on an event-by-event basis. The pseudorapidity dependent detector differences are taken into account using Singular Value Decomposition. This is followed by equalization of calorimeter energies to even out non-physical negatives after underlying event subtraction.

\section{Nuclear modification factor}

One of the most studied experimental signature of energy loss of partons in the hot and dense medium is the nuclear modification factor $\left(R_{A A}\right)$. The nuclear modification factor for particles
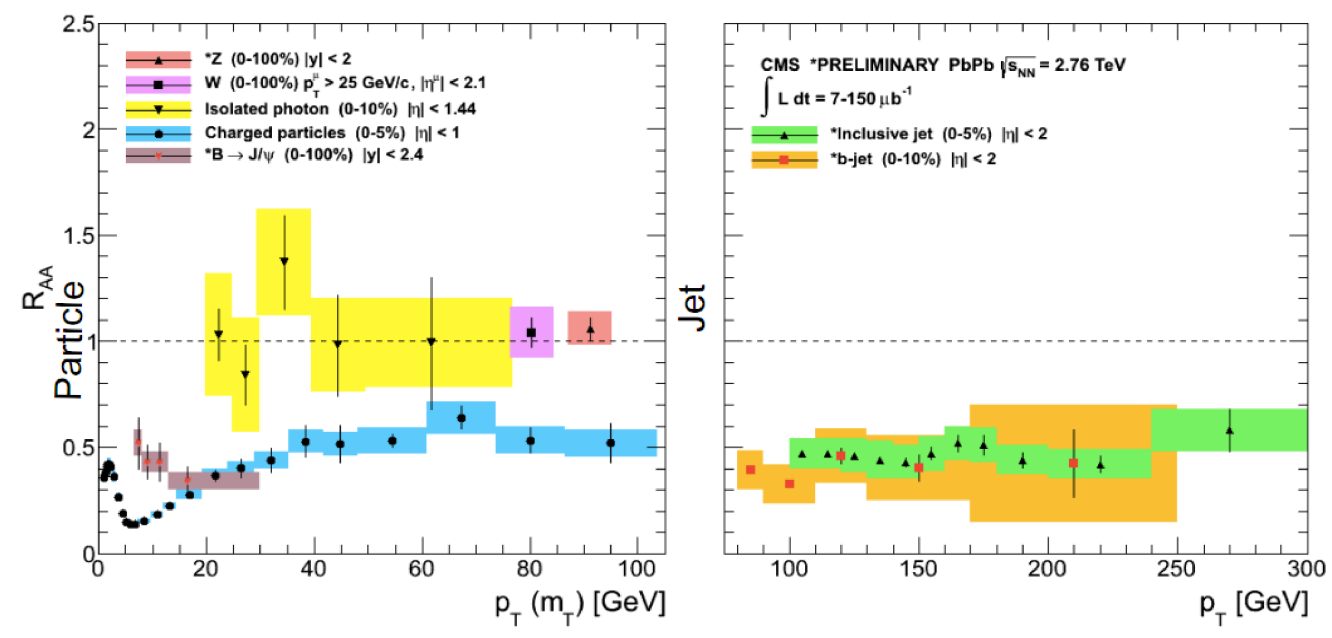

Figure 1: Nuclear Modification factor for charged particles, b-quarks separated from secondary $J / \psi$ particles, isolated photons, $W^{ \pm}$and $Z^{0}$ are shown in left panel. In right panel the $R_{A A}$ for fully reconstructed anti- $k_{T}$ jets and b-jets in $\mathrm{PbPb}$ collisions at $\sqrt{s_{N N}}=2.76 \mathrm{TeV}$. Statistical uncertainities are shown by verticle lines and systematic uncertainties are presented in shaded boxes around the data points.

or jets can be expressed by the ratio of measured yield in AA collisions to that in pp collision 
and scaled appropriately with the average number of nucleon-nucleon binary collisions $\left\langle N_{\text {coll }}\right\rangle$ calculated from Glauber model [15].

$$
R_{A A}=\frac{d N^{A A} / d p_{T}}{\left\langle N_{\text {coll }}\right\rangle d N^{p p} / d p_{T}},
$$

If the value of $R_{A A}=1$ suggests no in-medium modification of jets in $\mathrm{PbPb}$ collisions as compared to pp collisions. The measured $R_{A A}<1$ suggests parton energy loss and modification of jets in medium formed in $\mathrm{PbPb}$ collisions. Figure 1 shows the nuclear modification factor for different particles (in left panel) and for fully reconstructed anti- $k_{T}$ jets (in right panel) for $\mathrm{PbPb}$ collisions at $\sqrt{s_{N N}}=2.76 \mathrm{TeV}$. The charged particles [16] (mostly originating from the light flavor quarks and gluons) and b-quarks inferred from secondary $J / \psi$ [17] shows a suppression of yields in central $\mathrm{PbPb}$ collisions. Interestingly, the suppression factor for the charged particles and heavy-quark are of similar level indicating the independence of energy loss mechanism on the flavor of the parton. Also shown are the $R_{A A}$ for the isolated photons (0-10\%) [18], $W^{ \pm}$[19] and $Z^{0}$ [20] bosons (0$100 \%)$ at $\sqrt{s_{N N}}=2.76 \mathrm{TeV} \mathrm{PbPb}$ collisions. The above mentioned particles do not interact strongly with the medium formed in heavy-ion collisions and hence considered to be remained unmodified during their passage through medium. As expected there is no suppression in yields observed for these particles in $\mathrm{PbPb}$ collisions with respect to the measured yields in the pp collisions further validating the nucleon-nucleon binary collision scaling and these particles can be used as direct probes to quantify the energy loss in $\mathrm{PbPb}$ collisions. The left panel shows the comparison of $R_{A A}$ for the inclusive jets and from b-jets. Within the measured range of $p_{T}$ the suppression of yields are similar for inclusive and $b$-jets emphasizing the flavor independence of energy loss mechanism in the most central $\mathrm{PbPb}$ collisions.
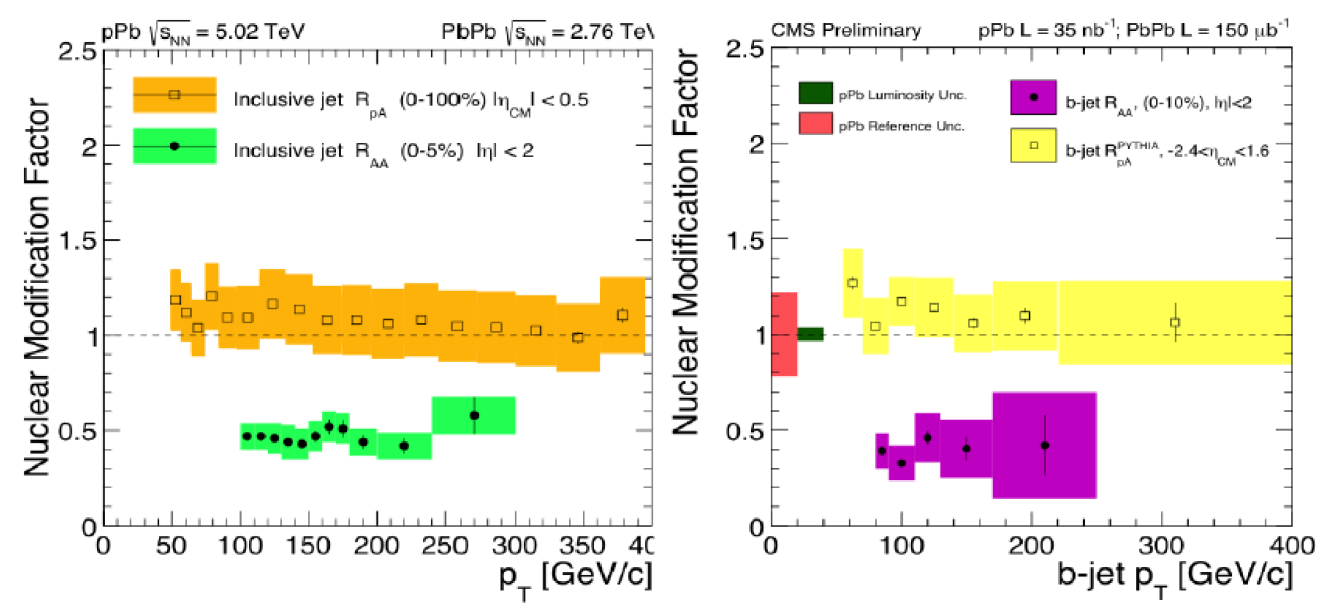

Figure 2: Nuclear Modification factor for inclusive jets (left) compared between $0-5 \% \mathrm{PbPb}$ and $0-100 \%$ $\mathrm{pPb}$ collisions. Comparison of $R_{A A}$ and $R_{p A}^{P Y T H I A}$ for b-jet (right) are shown for $0-10 \% \mathrm{PbPb}$ and for $\mathrm{pPb}$ collisions. The shaded boxes around the points represent the systematic uncertainties. The luminosity uncertainties are shown in shaded boxes at 1 in the left panel.

To understand the effect of medium on the energy loss of the probes we need to first understand the modification of these probes in the $\mathrm{pPb}$ collisions, where only initial state effects comes into 
picture. Figure 2 shows the comparison of inclusive jet (left panel) and b-jet (right panel) $R_{A A}$ and $R_{p A}$ in (0-5\%) $\mathrm{PbPb}$ and (0-100\%) $\mathrm{pPb}$ at $\sqrt{s_{N N}} 2.76$ and $5.02 \mathrm{TeV}$, respectively. The $\mathrm{pPb}$ jets (left panel) show minimal enhancement at low jet $p_{T}$ in comparison to a pp reference extrapolated from CMS measurements at $7 \mathrm{TeV}[21,22]$. The b-jets (right panel) that are tagged using distributions of the secondary vertex displacement compared to a reference of PYTHIA [23] event generated b-jets embedded into HIIJING [24]. The absence of suppression in inclusive and b-jet yield in $\mathrm{pPb}$ collisions indicate that the observed suppression in the central $\mathrm{PbPb}$ collisions is not attributed to the initial state effects in the observed kinematic range.

\section{Isolated photon-jet measurements}

Study of isolated photon-jet events can act as a probe to quantify the energy loss of the jets in the strongly interacting medium. These events are measured by requiring one of the partners of the jet to be an isolated photon, separated in azimuthal angle difference $\Delta \phi_{J \gamma}>\frac{7}{8} \pi$ from the second jet partner [25, 26]. This criteria was applied to suppress contribution from background jets as well as photon +2 -jets events. For these selected pairs we study the momentum fraction, $x_{j \gamma}($ $=p_{T}^{\text {Jet }} / p_{T}^{\gamma}$ ), between the reconstructed jet and its associated isolated photon and a quantity called $R_{j \gamma}$ which denotes the fraction of those isolated photons that are associated to a partner jet above $30 \mathrm{GeV} / c$. Figure 3 shows the $\left\langle x_{j \gamma}\right\rangle$ (left) and $R_{j \gamma}$ (right) are studied as a function of number
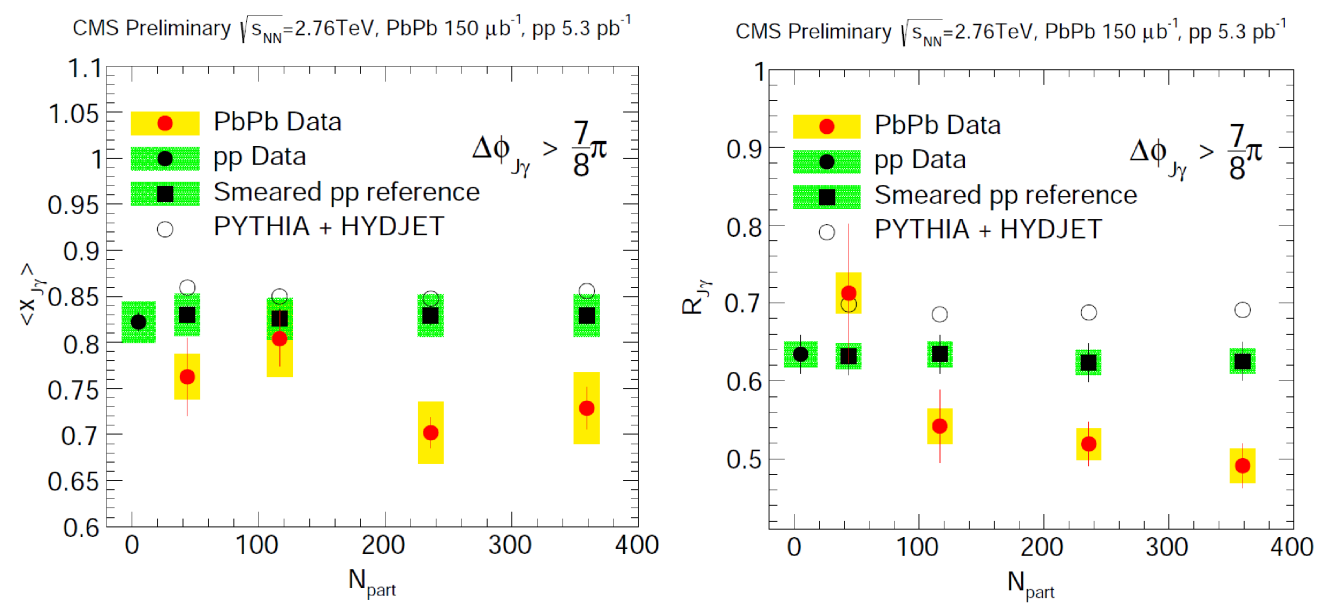

Figure 3: Comparison of $\left\langle x_{j \gamma}\right\rangle$ and $R_{j \gamma}$ in $\mathrm{pp}$ and $\mathrm{PbPb}$ collisions. The momenta of jets in pp were smeared by relative jet energy resolution to be used as the reference of each centrality bin. The shaded boxes represent the systematic uncertainty while the line through the points represent the statistical uncertainty.

of participating nucleons $\left(N_{\text {part }}\right)$ obtained from a Glauber model calculation which can be closely related to the measured centrality in heavy-ion collisions. For the comparison, also shown are the corresponding $\left\langle x_{j \gamma}\right\rangle$ and $R_{j \gamma}$ for pp collisions (black circle) and from the pp events generated from PYTHIA and embedded in $\mathrm{PbPb}$ events simulated by HYDJET [27]. The pp data (black square) is smeared to account for the jet resolution difference in $\mathrm{pp}$ and $\mathrm{PbPb}$ collisions when compared with $\mathrm{PbPb}$ data. The $\left\langle x_{j \gamma}\right\rangle$ are significantly lower in $\mathrm{PbPb}$ collision data for larger $N_{\text {part }}$ values as compared to smeared pp reference data which shows no dependence on $N_{\text {part }}$ in the 
studied $p_{T}$ range. The $R_{j \gamma}$ is lower by $01-0.2$ in central $\mathrm{PbPb}$ collisions than pp collisions, which indicates the larger fraction of jets lost energy and fell below $30 \mathrm{GeV} / c$.

\section{Jet shapes and fragmentation function}

To understand the mechanism of the energy loss by these energetic jets, it is very important to look at the constituents of these jets and study in detail their properties. These studies can be done experimentally by looking at the modifications in the transverse direction, i.e. jet shapes, as well as looking at the fragmentation pattern of the jet constituents in their momentum space. Both these analyses were performed using charged particles whose momenta can be measured with a very good precision in CMS tracker. Jet shapes can be defined as the transverse momentum fraction, $\rho(r)$, carried by charged particles as function of their distance $r=\sqrt{\Delta \eta^{2}+\Delta \phi^{2}}$ from the jet axis [?]. Figure 4 top panels shows the jet shape results for $\mathrm{PbPb}$ collisions (solid circle) in

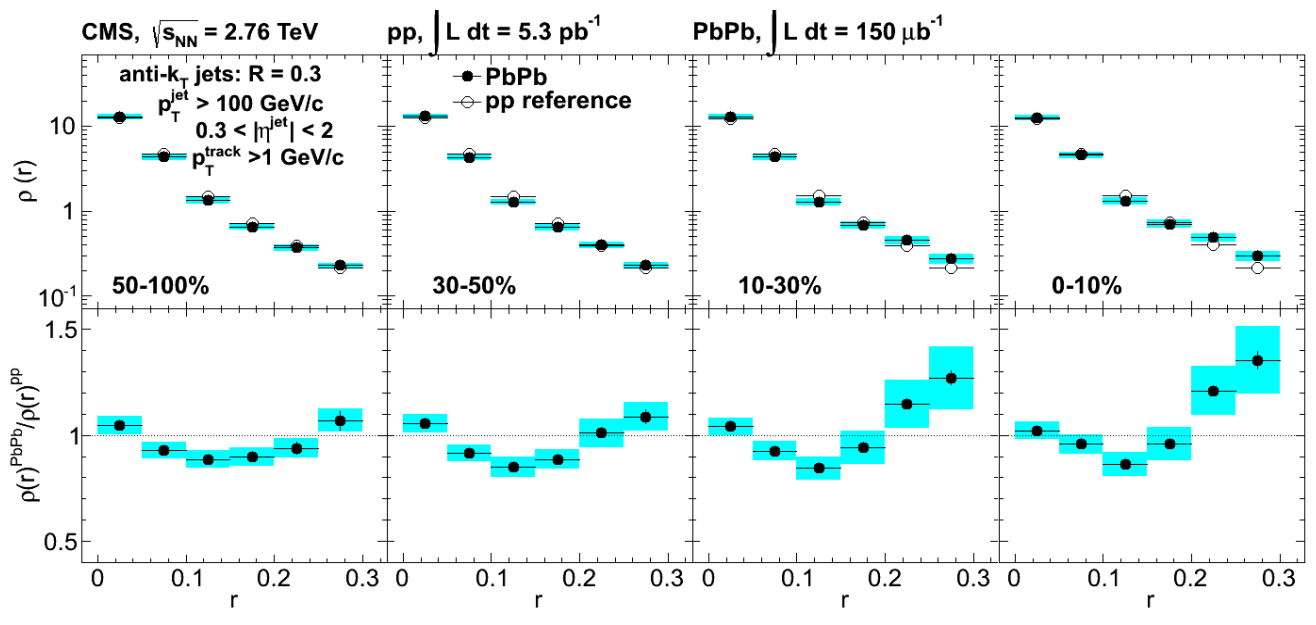

Figure 4: Differential jet shapes in $\mathrm{PbPb}$ collisions (solid circle) and pp reference shapes (open circle) are shown in four $\mathrm{PbPb}$ centrality intervals. Each spectrum is normalized to an integral of unity. The shaded regions represent the systematic uncertainties, with statistical uncertainties too small to be visible. Bottom row: Jet shape nuclear modification factors $\rho(r)^{P b P b} / \rho(r)^{p p}$. The error bars show the statistical uncertainties, and the shaded boxes indicate the systematic uncertainties.

four different collision centrality. For comparison, also shown are the pp reference jet shapes (open circle). The pp jet shape results are constructed taking into account the difference of the jet energy resolution and of the jet $p_{T}$ spectrum between the two different colliding systems. Bottom panels show the ratio between jet shapes measured in $\mathrm{PbPb}$ collisions and pp collisions. In peripheral collisions the jet shapes are very similar between $\mathrm{pp}$ and $\mathrm{PbPb}$ collisions. The depletion in yield at intermediate radii, $0.1<r<0.2$, and an excess of yield at large radii, $r>0.2$ is observed. This excess indicates that the energy that the jets lose in the medium is redistributed at larger distances from the jet axis outside the jet cone. The hadronization pattern of constituent particles in a jet can be understood by studying the jet fragmentation functions. The jet fragmentation function is defined as a function of the variable $\xi$

$$
z=\frac{p_{\|}^{\text {track }}}{p^{\text {Jet }}} ; \xi=\ln \frac{1}{z}
$$




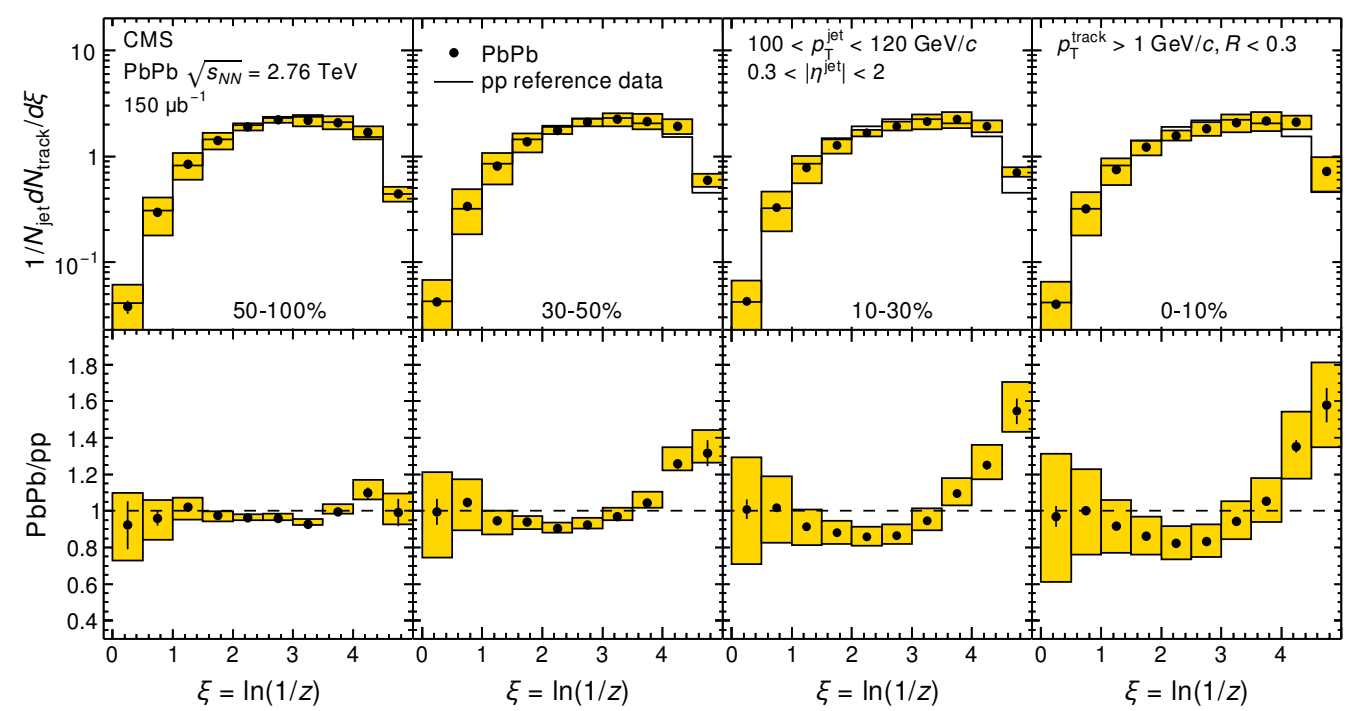

Figure 5: The $\mathrm{PbPb}$ fragmentation function in bins of centrality (increasing from left to right) overlaid with pp reference data. Jets have $100<p_{T}<120 \mathrm{GeV} / c$, and tracks have $p_{T}>1 \mathrm{GeV} / c$. Bottom panels show the ratio of each $\mathrm{PbPb}$ fragmentation function to its pp reference. Error bars are statistical, and shaded boxes show the systematic uncertainty.

For this study the charged particle tracks of $p_{T}>1 \mathrm{GeV} / c$ in the cone radius of 0.3 around the reconstructed jet axis are used to measure fragmentation functions. These measurements were done for different jet $p_{T}$ selections. Figure 5 (top) shows the fragmentation function for $\mathrm{PbPb}$ collisions (solid circles) and pp collisions (histograms) [29]. The bottom panels show the ratio of jet fragmentation functions from $\mathrm{PbPb}$ collisions to pp collisions. For the most central collisions, an enhancement of low $p_{T}$ particles, which corresponds to high $z$ values, is observed. There is suppression in the intermediate $p_{T}$ particles. Very high $p_{T}(\sim 35 \mathrm{GeV} / c)$ particles, which corresponds to very low $z$ values, are unmodified even in central collisions.

\section{Momentum flow in unbalanced jets}

The momentum flow for the charged particles in $\mathrm{pp}$ and $\mathrm{PbPb}$ collisions is performed on very high momentum jets in order to account for the missing energy which is observed in dijet asymmetry results [30]. For this analysis we look at the charged particles with $p_{T}>0.5 \mathrm{GeV} / c$ for highly asymmetric jets $\left(A_{J}>0.22\right)$ in momentum phase space. This large $A_{J}$ selection, which enhances the fraction of jets having undergone significant energy loss in the medium in $\mathrm{PbPb}$ collisions as compared to the results from pp collisions. The overall momentum balance in dijet events can be obtained from the projection of the $p_{T}$ of the reconstructed charged particles onto an axis in the azimuthal plane $\left(\phi_{\text {Dijet }}\right)$. For each event, this projection was calculated as,

$$
\not p_{T}^{\|}=\sum_{i}-p_{T}^{i} \cos \left(\phi_{i}-\phi_{D i j e t}\right)
$$

The momentum flow of charged particles is studied in the angular rings of $\Delta R=\sqrt{\Delta \phi_{T r k, \text { Jet }}^{2}+\Delta \eta_{T r k, J e t}^{2}}$ 


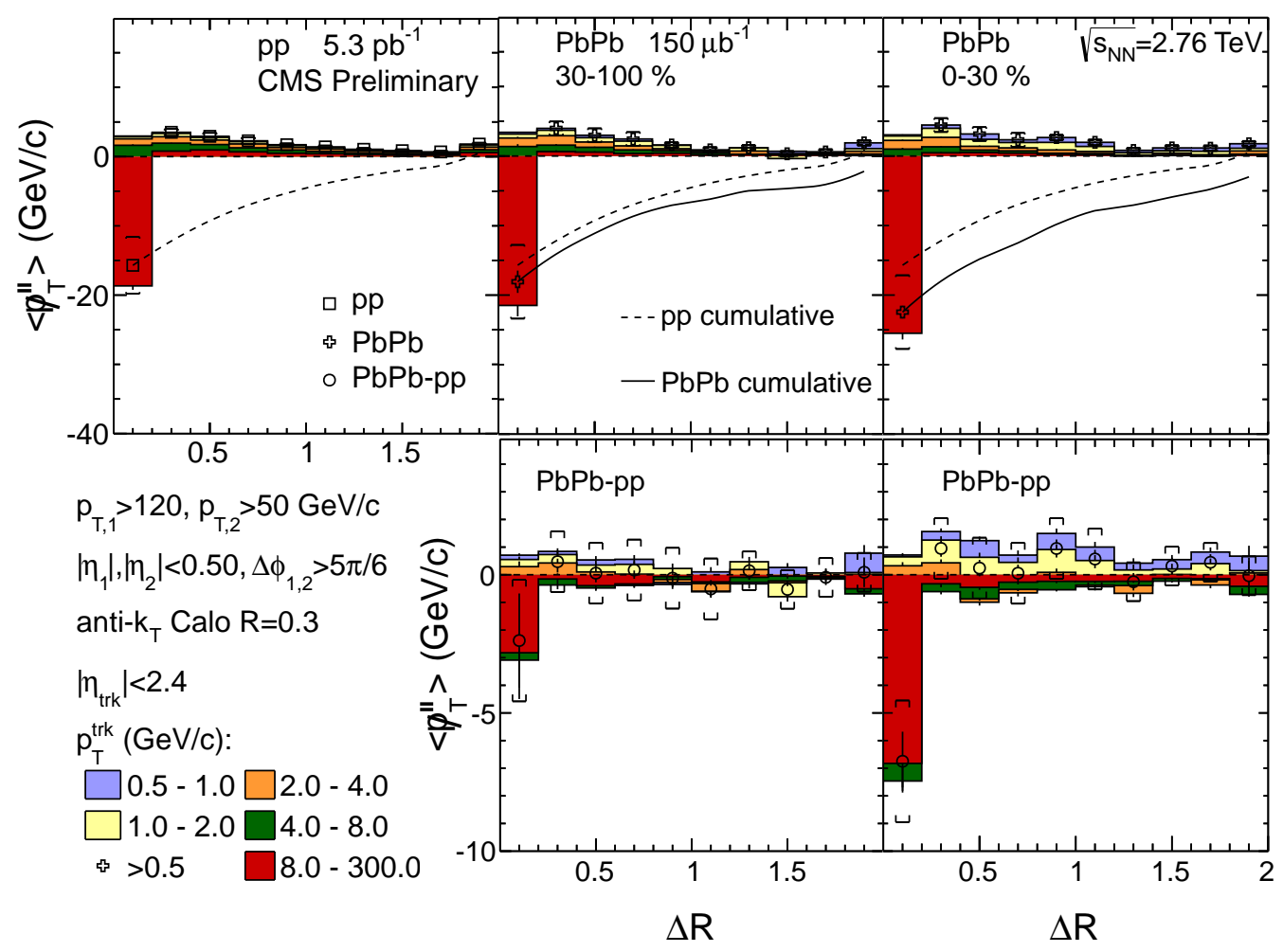

Figure 6: Upper panels : Differential missing $p_{T}$ distribution for pp, 30-100\% and 0-30\% $\mathrm{PbPb}$ data for various $p_{T}$ ranges (colored histograms), as a function of $\Delta R$

. Also shown are the total $<p_{T}>$ for pp (open square) and $\mathrm{PbPb}$ data (cross symbol). Dashed lines indicate the cumulative $\left.<p_{T}\right\rangle$ for events with large dijet asymmetry $\left(A_{J}>0.22\right.$. Bottom panels : Difference between $\mathrm{PbPb}$ and pp where the bars ans brackets represent statistical and systematic uncertainties respectively.

in pp and $\mathrm{PbPb}$ collisions. Figure 6, top left panel shows the $\Delta R$ dependence of missing $p_{T}$ for $\mathrm{pp}$ collisions, middle panel shows the results for peripheral $30-100 \% \mathrm{PbPb}$ collisions and right panel shows the results for central $0-30 \% \mathrm{PbPb}$ collisions. The bottom middle panel and right panel shows the difference in yields between peripheral and central $\mathrm{PbPb}$ collisions with that from $\mathrm{pp}$ collisions. The charged particle momentum flow distributions for $\mathrm{PbPb}$ and $\mathrm{pp}$ collisions are shown for different $p_{T}$ ranges, with red being the highest $p_{T}>8 \mathrm{GeV} / c$, and light blue indicating the lowest for $0.5<p_{T}<1.0 \mathrm{GeV} / c$ range. Larger imbalance is observed in $0-30 \% \mathrm{PbPb}$ collisions for charged particle with $p_{T}>2 \mathrm{GeV} / c$ at low $\Delta R(<0.2)$ when compared with the peripheral and pp collision results. As the $\Delta R$ is increased, this missing $p_{T}$ is balanced by low $p_{T}$ particles up to a very large $\Delta R$ of 2 . Also shown in the figure are the cumulative distribution for missing $p_{T}$ for $\mathrm{pp}$ (dashed curve) and central $\mathrm{PbPb}$ (solid curve). The asymmetry in dijet production in pp and $\mathrm{PbPb}$ have different causes, still the cumulative momentum flow of charged particles as a function of angular distribution are very similar for $\mathrm{PbPb}$ and $\mathrm{pp}$ collisions in the present $p_{T}$ ranges. 


\section{Conclusion}

The CMS experiment has shown various signatures of the jet energy loss in $\mathrm{PbPb}$ collisions at $\sqrt{s_{N N}}=2.76 \mathrm{TeV}$. The nuclear modification factor for inclusive jets and b-jets are presented for central $\mathrm{PbPb}$ collisions. The suppression factor for light quark jets and heavy quark jets are measured for $\mathrm{PbPb}$ collisions and found to be similar in the studied jet $p_{T}$ ranges. The inclusive and b-tagged jet yields in $\mathrm{pPb}$ collisions at $\sqrt{s_{N N}}=5.02 \mathrm{TeV}$ are systematically measured as not suppressed when compared to an extrapolated pp reference. Isolated photon jet results for transverse momentum ratio, $x_{j \gamma}$, and the fraction of photons with an associated jet, $R_{j \gamma}$, for different collision centralities. The $R_{j \gamma}$ central $\mathrm{PbPb}$ collisions was lower than pp collisions by $0.1-0.2$, which indicates a large fraction of jets lose energy and end up below $30 \mathrm{GeV} / c$. Jet shapes and jet fragmentation function results show an excess of low $p_{T}$ particles and no modification for the high $p_{T}$ particles in a jet. The momentum flow of tracks in unbalanced dijet pairs in both $\mathrm{PbPb}$ and $\mathrm{pp}$ collisions quantify the energy loss mechanism. There is about $35 \mathrm{GeV} / c$ of $p_{T}$ missing from the most quenched jets and the loss in $p_{T}$ is recovered by low $p_{T}<2 \mathrm{GeV} / c$ tracks upto $\Delta R$ of 2 units around the dijet axis. The angular distribution of the momentum flow of charged particles is found to be similar in $\mathrm{pp}$ and $\mathrm{PbPb}$ collisions, while the fragmentation is inherently different between $\mathrm{pp}$ and central $\mathrm{PbPb}$ collisions. In summary, these comprehensive results for jet production in heavyion collisions from CMS experiment has provided inputs for theoretical descriptions of energy loss models and established more precise experimental measurements for the energy loss mechanism in the dense medium formed in heavy-ion collisions.

\section{References}

[1] J. D. Bjorken, FERMILAB-PUB-82-059-THY.

[2] I. Arsene et. al. (BRAHMS Collaboration), Nucl. Phys. A 757, (2005), 1.

[3] B. B. Back et. al. (PHOBOS Collaboration), Nucl. Phys. A 757, (2005), 28.

[4] J. Adams et. al. (STAR Collaboration), Nucl. Phys. A 757, (2005), 102.

[5] K. Adcox et. al. (PHENIX Collaboration), Nucl. Phys. A 757, (2005), 184.

[6] S. Chatrchyan et. al. (CMS Collaboration), JINST 3, (2008), S08004.

[7] S. Chatrchyan et. al. (CMS Collaboration), Phys. Rev. C 84, (2011), 024906.

[8] Y. L. Dokshitzer and D. E. Kharzeev, Phys. Lett. B 519, (2001), 199.

[9] CMS Collaboration, CMS-PAS-PFT-09-001, (2009), [http://cds.cern.ch/record/1194487].

[10] M. Cacciari, G. P. Salam and G. Soyez, Eur. Phys. J. C 72, (2012), 1896.

[11] M. Cacciari, G. P. Salam and G. Soyez, JHEP 04, (2013), 063.

[12] O. Kodolova, I. Vardanian, A. Nikitenko et. al, Eur. Phys. J C 50, (2007), 117.

[13] S. Chatrchyan et. al. (CMS Collaboration), JINST 8, (2013), P04013.

[14] CMS Collaboration, CMS-DP-2013-018, (2013), [http://cds.cern.ch/record/1558644].

[15] M. L. Miller, K. Reygers, S. J. Sanders and P. Steinberg, Ann. Rev. Nucl. Part. Sci.57, (2007), 205. 
[16] S. Chatrchyan et. al. (CMS Collaboration), Eur. Phy. J. C 72, (2012), 1945.

[17] S. Chatrchyan et. al. (CMS Collaboration), CMS-PAS-HIN-12-014, (2012), [http://cdsweb.cern.ch/record/1472735].

[18] S. Chatrchyan et. al. (CMS Collaboration), Phys. Lett. B 710, (2012), 256.

[19] S. Chatrchyan et. al. (CMS Collaboration), Phys. Lett. B 715, (2012), 66.

[20] S. Chatrchyan et. al. (CMS Collaboration), CMS-PAS-HIN-12-008, (2012), [http://cdsweb.cern.ch/record/1472723].

[21] S. Chatrchyan et. al. (CMS Collaboration), Phys. Rev. Lett. 107, (2011), 132001.

[22] S. Chatrchyan et. al. (CMS Collaboration), Phys. Rev. D 87, (2013), 112002.

[23] T. Sjostrand, S. Mrenna and P. Z. Skands, JEHP 0605, (2006), 206.

[24] M. Gyulassy and X. N. Wang, Comput. Phys. Commun. 83, (1994), 307.

[25] S. Chatrchyan et. al. (CMS Collaboration), Phys. Lett. B 718, (2013), 773-794.

[26] CMS Collaboration, CMS-PAS-HIN-13-006, http://cds.cern.ch/record/1631991], (2013).

[27] I. Lokhtin, A. Snigirev, Eur. Phys. J. C 45, (2006), 211-217.

[28] S. Chatrchyan et. al. (CMS Collaboration), Phys. Lett. B 730, (2014), 243.

[29] S. Chatrchyan et. al. (CMS Collaboration), Phys. Rev. C 90, (2014), 024908.

[30] CMS Collaboration, CMS-PAS-HIN-14-010, (2014), [http://cds.cern.ch/record/1703014]. 